\title{
Some existence results for solutions of differential inclusions with retardations
}

\author{
by L. H. Erbe* ${ }^{*}$ W. Krawcewicz* and Shaozhu Chen** (Edmonton)
}

\begin{abstract}
Using the topological transversality method of Granas we prove an existence result for a system of differential inclusions with retardations of the form $y^{\prime \prime} \in$ $F\left(t, y, y^{\prime}, \Phi(y)\right)$. The result is applied to the study of the existence of solutions to an equation of the trajectory of an $r$-stage rocket with retardations.
\end{abstract}

$\S 1$. Introduction. In a recent paper J. Duvallet [4] studied the following discontinuous two-point boundary value problem:

$$
\left\{\begin{array}{l}
x^{\prime \prime}(t)=f_{1}\left(t, x(t), x^{\prime}(t)\right)+\operatorname{sgn}(\psi(x(t))) f_{2}\left(t, x(t), x^{\prime}(t)\right), \\
x(0)=\alpha, \quad x(1)=\beta, \quad \psi(\alpha)<0<\psi(\beta),
\end{array}\right.
$$

where $f_{1}$ and $f_{2}$ are continuous mappings from $[0,1] \times \mathbb{R}^{n} \times \mathbb{R}^{n}$ to $\mathbb{R}^{n}$, and $\psi$ is a convex function of class $C^{2}$. This problem is related to the study of a rocket trajectory from one point to another which is supposed to drop a stage at a fixed altitude in-between. Under the conditions

$$
\begin{aligned}
\left\|g_{i}(t, x, y)-g_{i}\left(t, x^{\prime}, y^{\prime}\right)\right\| \leq K\left\|x-x^{\prime}\right\| & +L\left\|y-y^{\prime}\right\|, \\
& i=1,2, x, x^{\prime}, y, y^{\prime} \in \mathbb{R}^{n}, \\
D \psi(x) \cdot g_{i}(t, x, y) \geq 0, \quad i= & 1,2,
\end{aligned}
$$

where $g_{1}=f_{1}-f_{2}$ and $g_{2}=f_{1}+f_{2}, K / 8+L / 2<1$, and under an additional condition on the constants $K$ and $L$, Duvallet obtained the existence of a solution to (1.1).

In this paper we study similar discontinuous problems by reformulating (1.1) in the form of a differential inclusion and by applying the topological transversality method of Granas. The motivation for the use of this

1991 Mathematics Subject Classification: Primary 34B15.

Key words and phrases: boundary value problem, differential inclusion with retardations, topological transversality.

* Research supported by grants from NSERC Canada.

** On leave from: Department of Mathematics, Shandong University, Jinan, Shandong, People's Republic of China. 
method is its simplicity and the fact that we do not require any knowledge of algebraic or differential topology. It is clear that our results may also be obtained by using topological degree theory arguments. Despite the fact that solutions to the differential inclusion may not be solutions in the classical sense, our approach permits us to study a much larger class of discontinuous problems.

As an example of an application we obtain an existence result for an equation of the trajectory of an $r$-stage rocked with retardations.

The topological transversality method is based on finding a priori bounds on solutions to a family of differential inclusions, which leads us to a homotopy in the class of multivalued compact maps such that one end of this homotopy is an essential map. For more information concerning the topological transversality method we refer to [8], [3], [7], [15], [16], [5]. Our main result, Theorem (2.4), gives the existence of solutions to the following retarded differential inclusion:

$$
\left\{\begin{array}{l}
y^{\prime \prime}(t) \in F\left(t, y(t), y^{\prime}(t), y\left(g_{1}(t)\right), \ldots, y\left(g_{m}(t)\right)\right) \quad \text { for a.e. } t \in[0, T] \\
y(0)=a, \quad y(T)=b, \quad y(t)=\rho(t) \text { if } t<0
\end{array}\right.
$$

where:

$g_{i}:[0, T] \rightarrow \mathbb{R}, i=1, \cdots, m$, are continuous functions such that $g_{i}(t) \leq t$,

$\rho:[-\sigma, 0] \rightarrow \mathbb{R}^{n}$ is a continuous function such that $\rho(0)=a,-\sigma=$ $\min \left\{\inf g_{i}: i=1, \cdots, m\right\}$, and

$F:[0, T] \times \mathbb{R}^{n} \times \mathbb{R}^{n} \times \mathbb{R}^{n \cdot m} \rightarrow \mathcal{K}\left(\mathbb{R}^{n}\right)$ is a Carathéodory multifunction.

(Here $\mathcal{K}\left(\mathbb{R}^{n}\right)$ denotes the class of all nonempty compact convex subsets of $\mathbb{R}^{n}$.)

We introduce the conditions (A1)-(A3), and we use them to obtain $a$ priori bounds on solutions to

$\left(1.2_{\lambda}\right)$

$$
\left\{\begin{array}{l}
y^{\prime \prime}(t)-\varepsilon y(t) \in \lambda\left\{F\left(t, y(t), y^{\prime}(t), y\left(g_{1}(t)\right), \ldots, y\left(g_{m}(t)\right)\right)-\varepsilon y(t)\right\}, \varepsilon>0, \\
y(0)=a, \quad y(t)=b, \quad \lambda \in[0,1], \quad y(t)=\rho(t) \text { if } t<0,
\end{array}\right.
$$

and next by applying the topological transversality theorem we obtain the existence of a solution to (1.2). We note than in the case considered by Duvallet, a priori bounds on solutions to (1.1) follow from the condition (1); if $\alpha=K / 8+L / 2$ then every solution $y(t)$ to (1.1) satisfies $\|y(t)\|,\left\|y^{\prime}(t)\right\| \leq$ $C /(1-\alpha)$ where $C$ is a suitable constant. Therefore, in some sense, the results obtained in this paper can be considered as generalizing the results of Duvallet. We show that the "height" function $\psi(x)$ can be used in order to obtain the a priori bounds on the solution $y(t)$. Our example in $\S 3$ of the application of our technique gives us a more accurate description of the rocket's trajectory, since it takes into consideration the time of combustion and possible retardations due to reaction delays. In spite of those 
complications, our method permits us to obtain, under some additional, but reasonable conditions, the existence of solutions to that rocket problem.

We would like to emphasize that it is a common practice to study, instead of a nonlinear equation with discontinuities, the associated differential inclusion. The obtained solutions provide us with a possibility of studying discontinuous phenomena. We remark that retarded differential inclusions of first order were studied in [13]. We refer also to [19] and [2].

Suppose that $\mathbb{E}$ and $\mathbb{F}$ are Banach spaces and let $X$ be a subset of $\mathbb{E}$. A multivalued map $\Gamma: X \rightarrow \mathcal{K}(Y)$, where $Y \subset \mathbb{F}$ and $\mathcal{K}(Y)$ denotes the class of all nonempty, compact and convex subsets of $Y$, is called upper semi-continuous (u.s.c.) if $\{x \in X: \Gamma(x) \subset U\}$ is an open subset of $X$ for any open $U$ in $Y . \Gamma$ is said to be compact if $\Gamma(X)=\bigcup\{\Gamma(x): x \in X\}$ is relatively compact in $Y$. We refer to [1] for some additional facts concerning multifunctions.

Let $K$ be a convex subset of the Banach space $\mathbb{E}$. For any bounded closed subsets $A$ and $B$ of $K$ such that $B \subset A$, let $\mathcal{C}_{K}(A, B)$ denote the set of all multivalued maps $\Gamma: A \rightarrow \mathcal{K}(K)$ such that (i) $\Gamma$ is a compact u.s.c. map, and (ii) $x \notin \Gamma(x)$ for all $x \in B$.

For results concerning the topological transversality method and the notation used here we refer to [5]. For other facts and additional developments, we refer to [3], [7], [12], [15], [16].

In this paper we study differential inclusions of the form

(*) $\quad y^{\prime \prime}(t) \in F\left(t, y(t), y^{\prime}(t), \Phi(y)(t)\right), \quad$ where $y:[0, T] \rightarrow \mathbb{R}^{n}$,

using the topological transversality method. A boundary value problem for the inclusion $(*)$ can be reformulated under some additional hypotheses as a fixed-point problem involving some multivalued map $\mathcal{F}$. In order to be able to use the topological transversality method we need to know that $\mathcal{F}$ is a completely continuous multivalued map, i.e. $\left.\mathcal{F}\right|_{X} \in \mathcal{C}$ for every bounded set $X$. The assumption that $F$ is a Carathéodory multifunction will guarantee that this is the case (cf. [17], [18]). For additional information and definitions, we refer to [5].

$\S 2$. Existence results for differential inclusions. Let $F:[0, T] \times$ $\mathbb{R}^{n} \times \mathbb{R}^{n} \times \mathbb{R}^{n \cdot m} \rightarrow \mathcal{K}\left(\mathbb{R}^{n}\right)$ be a Carathéodory multifunction. Suppose that $g_{i}:[0, T] \rightarrow \mathbb{R}, i=1, \ldots, m$, are continuous functions such that $g_{i}(t) \leq t$. We put $-\sigma=\min \left\{\inf g_{i}: i=1, \ldots, m\right\}$ and suppose that $-\sigma<0$. Let $\rho:[-\sigma, 0] \rightarrow \mathbb{R}^{n}$ be a given continuous function such that $\rho(0)=a$.

We will study the existence of solutions to the system of retarded differential inclusions

$$
\left\{\begin{array}{l}
y^{\prime \prime}(t) \in F\left(t, y(t), y^{\prime}(t), y\left(g_{1}(t)\right), \ldots, y\left(g_{m}(t)\right)\right) \quad \text { for a.e. } t \in[0, T] \\
y(0)=a, \quad y(T)=b
\end{array}\right.
$$


where we suppose that $y(t)=\rho(t)$ if $t<0$.

Before we give the fixed-point reformulation of the problem (2.1), let us introduce the following spaces:

$$
\begin{gathered}
C_{a}:=\left\{y \in C\left([0, T] ; \mathbb{R}^{n}\right): y(0)=a\right\}, \quad C:=\left[C\left([0, T] ; \mathbb{R}^{n}\right)\right]^{2+m}, \\
C_{1}:=C_{a} \times C\left([0, T] ; \mathbb{R}^{n}\right) .
\end{gathered}
$$

We define the operator $\Phi: C_{a} \rightarrow\left[C\left([0, T] ; \mathbb{R}^{n}\right)\right]^{m}$ by

$$
\Phi(u)=\left(\Phi_{1}(u), \ldots, \Phi_{m}(u)\right), \quad u \in C_{a},
$$

where

$$
\Phi_{i}(u)(t)= \begin{cases}u\left(g_{i}(t)\right) & \text { if } g_{i}(t) \geq 0, \\ \rho\left(g_{i}(t)\right) & \text { if } g_{i}(t)<0,\end{cases}
$$

for $i=1, \ldots, m$. Let us remark that if we endow the space $\left[C\left([0, T] ; \mathbb{R}^{n}\right)\right]^{m}$ with the norm $\left\|\left(u_{1}, \ldots, u_{m}\right)\right\|_{0}:=\max \left\{\left\|u_{i}\right\|_{0} ; i=1, \ldots, m\right\}$ where $\|u\|_{0}=$ $\sup \{\|u(t)\|: t \in[0, T]\}$, then $\|\Phi(u)-\Phi(v)\|_{0} \leq\|u-v\|_{0}$ and $\Phi$ is a continuous map. Now we can reformulate the problem (2.1) as follows:

$$
\left\{\begin{array}{l}
y^{\prime \prime}(t) \in F\left(t, y(t), y^{\prime}(t), \Phi(y)(t)\right) \quad \text { for a.e. } t \in[0, T], \\
y(0)=a, \quad y(T)=b .
\end{array}\right.
$$

Since our method of proof applies to the problem (2.2) without making any reference to $(2.1)$, in what follows we will consider only the system (2.2), where we suppose that $\Phi: C_{a} \rightarrow\left[C\left([0, T] ; \mathbb{R}^{n}\right)\right]^{m}$ is a continuous map, bounded on bounded subsets of $C_{a}$. Therefore, we define the function $\eta: \mathbb{R}^{+} \rightarrow \mathbb{R}^{+}$by $\eta(M):=\sup \left\{\|\Phi(A)\|_{0}: A \subset C_{a},\|A\|_{0} \leq M\right\}$.

We are looking for a solution to $(2.2)$ in the class $H^{2}\left([0, T] ; \mathbb{R}^{n}\right)$. Let $\varepsilon>0$ be a sufficiently small number (we will make this more precise later) and define the operator $L: H^{2} \rightarrow L^{2} \times \mathbb{R}^{n} \times \mathbb{R}^{n}$, where $H^{2}:=H^{2}\left([0, T] ; \mathbb{R}^{n}\right)$, $L^{2}:=L^{2}\left([0, T] ; \mathbb{R}^{n}\right)$, by $L y=\left(y^{\prime \prime}-\varepsilon y, y(0), y(T)\right)$. It is well known that $L$ is an isomorphism. We put $X_{a b}=L^{2} \times\{a\} \times\{b\}$ and $Y_{a b}=L^{-1}\left(X_{a b}\right)$. Observe that the operator $j: H^{2} \rightarrow\left[C\left([0, T] ; \mathbb{R}^{n}\right)\right]^{2}$ defined by $j(u)=\left(u, u^{\prime}\right)$ is completely continuous and that $j\left(Y_{a b}\right) \subset C_{1}$. Thus we can consider the diagram

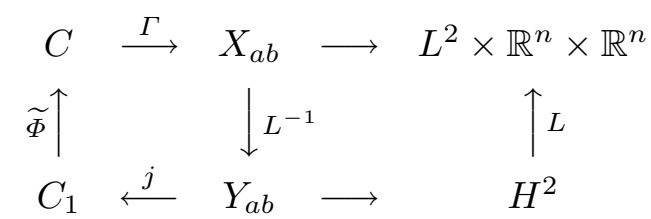

where $\widetilde{\Phi}(u, v)=(u, v, \Phi(u)),(u, v) \in C_{1}$, and

$$
\begin{aligned}
& \Gamma\left(u, v,\left\{w_{i}\right\}_{i=1}^{m}\right) \\
& =\left\{z \in L^{2}: z(t) \in \Gamma\left(t, u(t), v(t), w_{1}(t), \ldots, w_{m}(t)\right)-\varepsilon u(t)\right. \\
& \text { for a.e. } t \in[0, T]\} \times\{a\} \times\{b\} .
\end{aligned}
$$


The problem (2.2) is equivalent to the following fixed-point problem:

$$
(u, v) \in \mathcal{F}(u, v):=j \circ L^{-1} \Gamma(\widetilde{\Phi}(u, v)), \quad(u, v) \in C_{1} .
$$

We have supposed that $F$ is a Carathéodory multifunction, thus $\mathcal{F}$ is an u.s.c. multivalued map with convex and compact nonempty values which is completely continuous, i.e. $\mathcal{F}$ is upper semi-continuous and $\mathcal{F}(A)$ is relatively compact for every bounded subset $A \subset C$.

In order to solve (2.3) we consider the family of fixed-point problems

$$
(u, v) \in \lambda \mathcal{F}(u, v)+(1-\lambda)\left(y_{0}, y_{0}^{\prime}\right), \quad \lambda \in[0,1] .
$$

where $y_{0}$ is the unique solution to the equation $y^{\prime \prime}-\varepsilon y=0$ such that $y(0)=a, y(T)=b$.

Let us remark that the problem $\left(2.3_{\lambda}\right)$ is equivalent to the system of differential inclusions

$$
\left\{\begin{array}{l}
y^{\prime \prime}(t)-\varepsilon y(t) \in \lambda\left\{F\left(t, y(t), y^{\prime}(t), \Phi(y)(t)\right)-\varepsilon y(t)\right\} \\
y(0)=a, \quad y(T)=b, \quad \lambda \in[0,1] .
\end{array}\right.
$$

Therefore, in order to obtain the existence result for the system (2.2) it is a sufficient, by (2.5) in [5], to obtain a priori bounds on solutions to $\left(2.2_{\lambda}\right)$, for $\lambda \in[0,1]$.

Suppose that there is given a function $\psi: \mathbb{R}^{n} \rightarrow \mathbb{R}$ of class $C^{2}$ such that $\psi$ is convex and coercive, i.e. $\psi(x) \rightarrow \infty$ as $\|x\| \rightarrow \infty$. This implies that for every $R \in \mathbb{R}$ the set $\varphi^{-1}(-\infty, R]$ is bounded and we can put $\xi(R):=$ $\sup \left\{\|y\|: y \in \varphi^{-1}(-\infty, R]\right\}$. We will call $\psi$ a height function.

Now, let us introduce the following hypothesis:

(A1) There exists a constant $R>0$ such that if $\psi(a), \psi(b) \leq R$ and if $\psi\left(x_{0}\right)>R$ then for every $x_{0}^{\prime} \in \mathbb{R}^{n}$ such that $D \psi\left(x_{0}\right) \cdot x_{0}^{\prime}=0$ there exists $\delta>0$ such that

$$
\begin{aligned}
& \quad \underset{t \in[0, T]}{\operatorname{essinf} \inf \left\{D \psi(x) \cdot w+D^{2} \psi(x) x^{\prime} \cdot x^{\prime}:\right.} \\
& \left.\qquad w \in F\left(t, x, x^{\prime}, z\right),\|z\|<\widehat{\eta},\left(x, x^{\prime}\right) \in \mathcal{D}_{\delta}\right\}>0, \\
& \text { where } \widehat{\eta}:=\eta\left(\xi\left(\psi\left(x_{0}\right)\right)\right), \mathcal{D}_{\delta}:=\left\{\left(x, x^{\prime}\right) \in \mathbb{R}^{2 n}:\left\|x_{0}-x\right\|+\left\|x_{0}^{\prime}-x^{\prime}\right\|\right. \\
& <\delta\} .
\end{aligned}
$$

We also need the following Nagumo growth conditions:

(A2) There is a function $\varphi:[0, \infty) \rightarrow(0, \infty)$ such that $s / \varphi(s) \in L_{\mathrm{loc}}^{\infty}[0, \infty)$, $\int_{0}^{\infty}(s / \varphi(s)) d s=\infty$ and

$$
\left\|F\left(t, y, y^{\prime}, z\right)\right\| \leq \varphi\left(\left\|y^{\prime}\right\|\right)
$$

for a.e. $t \in[0, T]$ and all $\left(y, y^{\prime}, z\right) \in \mathcal{D}:=\left\{\left(x, x^{\prime}, w\right):\|x\| \leq\right.$ $\xi(R),\|w\| \leq \eta(\xi(R))\}$. 
(A3) There exist constants $K, \alpha>0$ such that

$$
\left\|F\left(t, y, y^{\prime}, z\right)\right\| \leq 2 \alpha\left(y \cdot w+\left\|y^{\prime}\right\|^{2}\right)+K
$$

for a.e. $t \in[0, T]$ and all $\left(y, y^{\prime}, z\right) \in \mathcal{D}, w \in F\left(t, y, y^{\prime}, z\right)$.

We observe that condition (A1), in the case of a continuous function and with the height function $\psi(x)$ replaced by $\|x\|^{2}$, reduces to the classical Nagumo-Hartman type condition. In [5] a similar condition to (A1) was studied (see also [10], [11], [12], [9], [7] where the scalar Carathéodory case was considered). Conditions (A2), (A3) are related to the usual NagumoBernstein growth conditions (cf. [14], [10], [7], [6] and the references therein).

In $\S 3$ we present an example of a problem with discontinuities for which conditions (A1)-(A3) reduce to classical Nagumo type conditions. However, this cannot be expected to occur when the function has more complicated discontinuities.

(2.4) Theorem. Suppose that $F:[0, T] \times \mathbb{R}^{n} \times \mathbb{R}^{n} \times \mathbb{R}^{n \cdot m} \rightarrow \mathcal{K}\left(\mathbb{R}^{n}\right)$ is a Carathéodory multifunction, and $\Phi: C_{a} \rightarrow\left[C\left([0, T] ; \mathbb{R}^{n}\right)\right]^{m}$ a continuous map which is bounded on bounded sets such that the hypotheses (A1)-(A3) are satisfied. Then the differential inclusion

$$
\left\{\begin{array}{l}
y^{\prime \prime}(t) \in F\left(t, y(t), y^{\prime}(t), \Phi(y)(t)\right) \quad \text { for a.e. } t \in[0, T], \\
y(0)=a, \quad y(T)=b
\end{array}\right.
$$

has at least one solution in $H^{2}\left([0, T] ; \mathbb{R}^{n}\right)$.

P r o of. In order to obtain the existence result we need a priori bounds on solutions of the system $\left(2.2_{\lambda}\right)$.

We observe that, since $\psi: \mathbb{R}^{n} \rightarrow \mathbb{R}$ is a convex $C^{2}$-function, $D^{2} \psi(x) x^{\prime} \cdot x^{\prime}$ $\geq 0$ for all $x, x^{\prime} \in \mathbb{R}^{n}$. This implies that for every $\lambda \in(0,1]$ the multifunction $\lambda F\left(t, y, y^{\prime}, z\right)+(1-\lambda) \varepsilon y$ also satisfies the assumption (A1) with the same constants $R, \delta$ and $\xi$. On the other hand, it is well known (see [5] or [10]) that there exists an $\varepsilon>0$, sufficiently small, such that the conditions (A2) and (A3), by Lemma (4.2) in [5], will still imply the a priori bounds on $\left\|y^{\prime}(t)\right\|$, where $y$ is a solution to $\left(2.2_{\lambda}\right)$, provided that $\|y(t)\| \leq \xi(R)$.

Suppose therefore that $y \in H^{2}\left([0, T] ; \mathbb{R}^{n}\right)$ is a solution to $\left(2.2_{\lambda}\right), \lambda \in$ $(0,1]$. We will show that $\|y(t)\| \leq \xi(R)$ for all $t \in[0, T]$. Suppose for contradiction that $\max \|y(t)\|>\xi(R)$. This implies that there exists a point $t_{0} \in(0, T)$ such that $\psi\left(y\left(t_{0}\right)\right)>R$. Since $\psi(a), \psi(b) \leq R$, the function $\gamma(t):=\psi(y(t))$ achieves its maximum at some point $t_{1} \in(0, T)$ and $\gamma\left(t_{1}\right)=$ $\max _{t \in[0, T]} \psi(y(t))>R$. Thus $\gamma^{\prime}\left(t_{1}\right)=D \psi\left(y\left(t_{1}\right)\right) \cdot y^{\prime}\left(t_{1}\right)=0$ and we can apply the assumption (A1) to conclude that there exists a $\delta>0$ such that

$$
\begin{aligned}
& \underset{t \in[0, T]}{\operatorname{ess} \inf \inf }\left\{D \psi(x) \cdot w+D^{2} \psi(x) x^{\prime} \cdot x^{\prime}:\right. \\
& \left.\quad w \in \lambda F\left(t, x, x^{\prime}, z\right)+(1-\lambda) \varepsilon y,\|z\|<\widehat{\eta},\left(x, x^{\prime}\right) \in \mathcal{D}_{\delta}\right\}>0,
\end{aligned}
$$


where $\widehat{\eta}=\eta\left(\xi\left(\gamma\left(t_{1}\right)\right)\right)$. Since $\left(y(t), y^{\prime}\left(t_{1}\right)\right) \rightarrow\left(y\left(t_{1}\right), y^{\prime}\left(t_{1}\right)\right)$ as $t \rightarrow t_{1}$, there exists a $\mu>0$ such that for almost every $t \in A_{\mu}:=\left\{t \in[0, T]:\left|t_{1}-t\right|<\mu\right\}$ we have

$$
\begin{aligned}
& \inf \left\{D \psi(y(t)) \cdot w+D^{2} \psi(y(t)) y^{\prime}(t) \cdot y^{\prime}(t):\right. \\
& \left.w \in \lambda F\left(t, x, x^{\prime}, z\right)+(1-\lambda) \varepsilon y,\|z\|<\widehat{\eta}\right\}>0 .
\end{aligned}
$$

But $\psi(y(t)) \leq \psi\left(y\left(t_{1}\right)\right)$, thus $\|y(t)\| \leq \xi\left(\psi\left(y\left(t_{1}\right)\right)\right)=: \xi_{1}$ and thus $\|\Phi(y)(t)\|$ $\leq \eta\left(\xi_{1}\right)=\widehat{\eta}$ and so

$$
\gamma^{\prime \prime}(t)=D \psi(y(t)) \cdot y^{\prime \prime}(t)+D^{2} \psi(y(t)) y^{\prime}(t) \cdot y^{\prime}(t)>0
$$

for a.e. $t \in A_{\mu}$. But this contradicts the maximum principle. This implies that $\|y(t)\| \leq \xi(R)$ for all $t \in[0, T]$.

As mentioned earlier, the estimates on the first derivative $\left\|y^{\prime}(t)\right\| \leq M_{1}$ are obtained by the Nagumo conditions (A2) and (A3), by applying Lemma (4.2) of [5].

Consider now a Carathéodory multifunction $F:[0, \infty) \times \mathbb{R}^{n} \times \mathbb{R}^{n} \times$ $\mathbb{R}^{n \cdot m} \rightarrow \mathcal{K}\left(\mathbb{R}^{n}\right)$ and let $\rho:(-\infty, 0] \rightarrow \mathbb{R}^{n}$ be a given bounded and continuous function such that $\rho(0)=a$. Suppose that $g_{i}:[0, \infty) \rightarrow \mathbb{R}, i=1, \ldots, m$, are continuous functions such that $g_{i}(t) \leq t$.

We will now study the system of retarded differential inclusions

$$
\left\{\begin{array}{l}
y^{\prime \prime}(t) \in F\left(t, y(t), y^{\prime}(t), y\left(g_{1}(t)\right), \ldots, y\left(g_{m}(t)\right)\right) \\
y(0)=a .
\end{array}\right.
$$

We refer to [11], [12], [7], [5] where these problems were also studied by similar methods. We suppose that the following conditions are satisfied. These conditions are the analogues of (A1)-(A3) for $t \in[0, \infty)$.

(B1) There exists a constant $R>0$ such that if $\psi(a) \leq R$ and if $\psi\left(x_{0}\right)>$ $R$ then for every $x_{0}^{\prime} \in \mathbb{R}^{n}$ such that $D \psi\left(x_{0}\right) \cdot x_{0}^{\prime}=0$ and for every compact subset $[0, T] \subset[0, \infty), T>0$ there exists a $\delta>0$ such that

$$
\begin{aligned}
& \underset{t \in[0, T]}{\operatorname{ess} \inf } \inf \left\{D \psi(x) \cdot w+D^{2} \psi(x) x^{\prime} \cdot x^{\prime}:\right. \\
& \left.\qquad w \in F\left(t, x, x^{\prime}, z\right),\|z\|<\widehat{\eta},\left(x, x^{\prime}\right) \in \mathcal{D}_{\delta}\right\}>0
\end{aligned}
$$

where $\widehat{\eta}:=\eta\left(\xi\left(\psi\left(x_{0}\right)\right)\right), \mathcal{D}_{\delta}:=\left\{\left(x, x^{\prime}\right) \in \mathbb{R}^{2 n}:\left\|x_{0}-x\right\|+\left\|x_{0}^{\prime}-x^{\prime}\right\|\right.$ $<\delta\}$ and $\psi$ denotes the height function.

(B2) There is a function $\varphi:[0, \infty) \rightarrow(0, \infty)$ such that $s / \varphi(s) \in L_{\mathrm{loc}}^{\infty}[0, \infty)$, $\int_{0}^{\infty}(s / \varphi(s)) d s=\infty$ and $\left\|F\left(t, y, y^{\prime}, z\right)\right\| \leq \varphi\left(\left\|y^{\prime}\right\|\right)$ for a.e. $t \in[0, \infty)$ and all $\left(y, y^{\prime}, z\right) \in \mathcal{D}:=\left\{\left(x, x^{\prime}, u\right):\|x\|,\|u\| \leq \xi(R)\right\}$. 
(B3) There exist constants $K, \alpha>0$ such that

$$
\left\|F\left(t, y, y^{\prime}, z\right)\right\| \leq 2 \alpha\left(y \cdot w+\left\|y^{\prime}\right\|^{2}\right)+K
$$

for a.e. $t \in[0, \infty)$ and all $\left(y, y^{\prime}, z\right) \in \mathcal{D}, w \in F\left(t, y, y^{\prime}, z\right)$.

(2.6) Theorem. Suppose that $F:[0, \infty) \times \mathbb{R}^{n} \times \mathbb{R}^{n} \times \mathbb{R}^{n \cdot m} \rightarrow \mathcal{K}\left(\mathbb{R}^{n}\right)$ is a Carathéodory multifunction such that the hypotheses (B1)-(B3) are satisfied. Then the differential inclusion (2.5) has a solution $y(t)$ in $H_{\mathrm{loc}}^{2}\left([0, \infty) ; \mathbb{R}^{n}\right)$. Moreover, if $\varphi \in L_{\mathrm{loc}}^{\infty}[0, \infty)$, then $y \in W^{2, \infty}\left([0, \infty) ; \mathbb{R}^{n}\right)$.

Pro of. We consider the family of differential inclusions

$$
\left\{\begin{array}{l}
y^{\prime \prime}(t) \in F\left(t, y(t), y^{\prime}(t), y\left(g_{1}(t)\right), \ldots, y\left(g_{m}(t)\right)\right) \\
y(0)=a, \quad y(N)=0,
\end{array}\right.
$$

where $N$ is a positive integer.

It follows from Theorem (2.4) that $\left(2.5_{N}\right)$ has a solution $y_{N} \in$ $H^{2}\left([0, N] ; \mathbb{R}^{n}\right)$. Moreover, for all $N \in \mathbb{N}$ the sequence $\left\{y_{N+k}\right\}_{k=1}^{\infty}$ restricted to the space $H^{2}\left([0, N] ; \mathbb{R}^{n}\right)$ is bounded and thus it contains a subsequence convergent in $C^{1}$-norm. Using a "diagonal method" of choosing successively convergent subsequences of $\left\{y_{N+k}\right\}_{k}$ in $C^{1}\left([0, N] ; \mathbb{R}^{n}\right)$, as $N \rightarrow \infty$, we find a subsequence $\left\{y_{N(k)}\right\}_{k}$ of $\left\{y_{N}\right\}_{N}$ such that there is a $C^{1}$-function $y:[0, \infty) \rightarrow \mathbb{R}^{n}$ such that $\left.\left.y_{N(k)}\right|_{[0, N]} \rightarrow y\right|_{[0, N]}$ in $C^{1}$-norm for all $N \in \mathbb{N}$. Using exactly the same arguments as in the proof of Theorem (6.1) in [5], we can show that $y(t)$ satisfies $(2.5)$ and $y \in H_{\mathrm{loc}}^{2}\left([0, \infty) ; \mathbb{R}^{n}\right)$.

Suppose that $\varphi \in L_{\mathrm{loc}}^{\infty}[0, \infty)$. Since

$$
\left\|F\left(t, y, y^{\prime}, \Phi(y)(t)\right)\right\| \leq \varphi\left(\left\|y^{\prime}\right\|\right)
$$

we have

$$
\left\|y^{\prime \prime}(t)\right\| \leq \varphi(\|y(t)\|) \leq \operatorname{ess} \sup \left\{\varphi(s): s \in\left[0,\left\|y^{\prime}\right\|_{0}\right]\right\}<\infty
$$

and this shows that $y \in W^{2, \infty}\left([0, \infty) ; \mathbb{R}^{n}\right)$.

§3. Application to an equation of the trajectory of an $r$-stage rocket with retardations. Suppose that $f_{j}:[0, T] \times \mathbb{R}^{n} \times \mathbb{R}^{n} \times \mathbb{R}^{n \cdot m} \rightarrow \mathbb{R}^{n}$, $j=0,1, \ldots, r-1$, are continuous functions and let $\psi: \mathbb{R}^{n} \rightarrow \mathbb{R}$ be a height function. We define

$$
f\left(t, y, y^{\prime}, u\right)=f_{0}\left(t, y, y^{\prime}, u\right)+\sum_{j=1}^{r-1} h_{j}(t, y) f_{j}\left(t, y, y^{\prime}, u\right)
$$

where the functions $h_{j}(t, y)$ are defined as follows:

(a) $\psi(a)<\alpha_{1}<\ldots<\alpha_{r-1}$. The constant $\alpha_{j}$ corresponds to the level of altitude at which the $j$ th stage is dropped. 
(b) $t_{1}, \ldots, t_{r-1}$ are positive constants such that $t_{1}+\ldots+t_{r-1} \leq T$. The constant $t_{j}$ corresponds to the time of combustion for the fuel in the $j$ th stage.

Let $H$ be the Heaviside function defined by $H(s)=0$ if $s<0$ and $H(s)=1$ if $s \geq 0$. We put

$$
h_{1}(t, y)=H\left(\max \left\{\psi(y)-\alpha_{1}, t-t_{1}\right\}\right), \quad t_{1}^{*}(y)=\inf \left\{t: h_{1}(t, y)=1\right\},
$$

and by induction, if $h_{m}(t, y)$ and $t_{m}^{*}(y)$ are already defined, we put for $m+1 \leq r-1$

$$
\begin{aligned}
h_{m+1}(t, y) & =H\left(\max \left\{\psi(y)-\alpha_{m+1}, t-\left(t_{m}^{*}(y)+t_{m+1}\right)\right\}\right), \\
t_{m+1}^{*}(y) & =\inf \left\{t: h_{m}(t, y)=1\right\} .
\end{aligned}
$$

(c) $t_{m}^{*}$ corresponds to the moment of time when the $m$ th stage is dropped. Moreover, $t_{m}^{*}$ depends continuously on $y$.

We consider the boundary value problem

$$
\left\{\begin{array}{l}
y^{\prime \prime}(t)=f\left(t, y(t), y^{\prime}(t), \Phi(y)(t)\right) \quad \text { for a.e. } t \in[0, T] \\
y(0)=a, \quad y(T)=b
\end{array}\right.
$$

where $\Phi(u)=\left(\Phi_{1}(u), \ldots, \Phi_{m}(u)\right)$ is the retardation operator associated with the retardations $g_{i}:[0, T] \rightarrow \mathbb{R}, g_{i}(t) \leq t, i=1, \ldots, m$, by the formula

$$
\Phi_{i}(u)= \begin{cases}u\left(g_{i}(t)\right) & \text { if } g_{i}(t) \geq 0, \\ a & \text { if } g_{i}(t)<0 .\end{cases}
$$

$\Phi$ represents the delays caused by reaction time.

In order to study the existence of solutions to (3.2) we consider the following differential inclusion which is associated to (3.2):

$$
\left\{\begin{array}{l}
y^{\prime \prime}(t) \in F\left(t, y(t), y^{\prime}(t), \Phi(y)(t)\right) \quad \text { for a.e. } t \in[0, T] \\
y(0)=a, \quad y(T)=b
\end{array}\right.
$$

where

$$
\begin{aligned}
& F\left(t, y, y^{\prime}, u\right) \\
& =\left[\underline{f}_{1}\left(t, y, y^{\prime}, u\right), \bar{f}_{1}\left(t, y, y^{\prime}, u\right)\right] \times \ldots \times\left[\underline{f}_{n}\left(t, y, y^{\prime}, u\right), \bar{f}_{n}\left(t, y, y^{\prime}, u\right)\right], \\
& \quad \underline{f}_{i}\left(t, y, y^{\prime}, u\right)=\liminf _{x \rightarrow y} f_{i}\left(t, x, y^{\prime}, u\right), \quad i=1, \ldots, n, \\
& \quad \bar{f}_{i}\left(t, y, y^{\prime}, u\right)=\limsup _{x \rightarrow y} f_{i}\left(t, x, y^{\prime}, u\right), \quad i=1, \ldots, n .
\end{aligned}
$$

Let us remark that the functions $f\left(t, y, y^{\prime}, u\right)$ and $\bar{f}\left(t, y, y^{\prime}, u\right)$ are finite and it is easy to check that $F\left(t, y, y^{\prime}, u\right)$ is a Carathéodory multifunction. For, let $\varepsilon=\left(\varepsilon_{1}, \ldots, \varepsilon_{r-1}\right)$ denote a multi-index, where $\varepsilon_{i}=0$ or 1 , and put

$$
g_{\varepsilon}\left(t, y, y^{\prime}, u\right)=f_{0}\left(t, y, y^{\prime}, u\right)+\sum_{j=1}^{r-1} \varepsilon_{j} f_{j}\left(t, y, y^{\prime}, u\right) \text {. }
$$


There exists a constant $c>0$ (related to the norm in $\mathbb{R}^{n}$ ) such that

$\left\|F\left(t, y, y^{\prime}, u\right)\right\| \leq c \max \left\{\left\|g_{\varepsilon}\left(t, y, y^{\prime}, u\right)\right\|: \varepsilon=\left(\varepsilon_{1}, \ldots, \varepsilon_{r-1}\right), \varepsilon_{i}=0\right.$ or 1$\}$.

Now we can define the function $\beta$ by

$$
\begin{aligned}
& \beta(r)=1+c \max \left\{\left\|g_{\varepsilon}\left(t, y, y^{\prime}, u\right)\right\|:\right. \\
& \left.\quad\left(t, y, y^{\prime}, u\right) \in[0, T] \times B_{r}, \varepsilon=\left(\varepsilon_{1}, \ldots, \varepsilon_{r-1}\right), \varepsilon_{i}=0 \text { or } 1\right\},
\end{aligned}
$$

where $B_{r}=\left\{\left(x, x^{\prime}, u\right):\left\|\left(x, x^{\prime}, u\right)\right\| \leq r\right\}$. By continuity of $g_{\varepsilon}$, the function $\beta:[0, \infty) \rightarrow(0, \infty)$ is continuous and thus $\beta, 1 / \beta \in L_{\mathrm{loc}}^{\infty}[0, \infty)$. It is evident that $\left\|F\left(t, y, y^{\prime}, u\right)\right\| \leq \beta\left(\left\|\left(y, y^{\prime}, u\right)\right\|\right)$, for all $\left(t, y, y^{\prime}, u\right) \in[0, T] \times$ $\mathbb{R}^{n} \times \mathbb{R}^{n} \times \mathbb{R}^{n \cdot m}$ and therefore $F$ satisfies the weak growth condition; hence $F$ is Carathéodory.

Let $R>0$ be a constant such that $R>\max \left\{\alpha_{r-1}, \psi(b)\right\}$. By definition, if $\psi(x)>R$ then $f\left(t, x, x^{\prime}, u\right)=f_{0}\left(t, x, x^{\prime}, u\right)+\sum_{j=1}^{r-1} f_{j}\left(t, x, x^{\prime}, u\right)$, thus the continuity of the functions $f_{0}, \ldots, f_{r-1}$ implies that for every $x \in \mathbb{R}^{n}$ such that $\psi(x)<R$ the multifunction $F$ is single-valued and $F\left(t, x, x^{\prime}, u\right)=$ $f\left(t, x, x^{\prime}, u\right)=f_{0}\left(t, x, x^{\prime}, u\right)+\sum_{j=1}^{r-1} f_{j}\left(t, x, x^{\prime}, u\right)$. Therefore, in this particular case, the hypothesis (A1) of $\S 3$ can be simplified to the following condition.

$(\mathrm{A} 1)^{\prime \prime} \quad$ If $\psi(x)>R$ then for every $x^{\prime} \in \mathbb{R}^{n}$ such that $D \psi(x) \cdot x^{\prime}=0$ and every $u$ such that $\|u\| \leq \xi(\psi(x))$ we have

$$
D \psi(x) \cdot f\left(t, x, x^{\prime}, u\right)+D^{2} \psi(x) x^{\prime} \cdot x^{\prime}>0 .
$$

We note that $(\mathrm{A} 1)^{\prime \prime}$ is somewhat weaker than the condition $(2)$ in $\S 1$, used in the paper of Duvallet (see [4]).

In order to apply Theorem (2.4) we suppose that the following Nagumo conditions are satisfied.

$(\mathrm{A} 2)^{\prime \prime} \quad$ There is a function $\varphi:[0, \infty) \rightarrow(0, \infty)$ such that $s / \varphi(s) \in$ $L_{\mathrm{loc}}^{\infty}[0, \infty), \int_{0}^{\infty}(s / \varphi(s)) d s=\infty$ and $\left\|F\left(t, y, y^{\prime}, z\right)\right\| \leq \varphi\left(\left\|y^{\prime}\right\|\right)$ for a.e. $t \in[0, T]$ and all $\left(y, y^{\prime}, z\right) \in \mathcal{D}:=\left\{\left(x, x^{\prime}, w\right):\|x\| \leq \xi(R)\right.$, $\|w\| \leq \xi(R)\}$.

$(\mathrm{A} 3)^{\prime \prime} \quad$ There exist constants $K, \alpha>0$ such that $\left\|F\left(t, y, y^{\prime}, z\right)\right\| \leq$ $2 \alpha\left(y \cdot w+\left\|y^{\prime}\right\|^{2}\right)+K$ for a.e. $t \in[0, T]$ and all $\left(y, y^{\prime}, z\right) \in \mathcal{D}, w \in$ $F\left(t, y, y^{\prime}, z\right)$.

Observe that in the particular case where all the functions $f_{i}, i=$ $0, \ldots, r-1$, satisfy the quadratic growth condition with respect to $y^{\prime}$, i.e.

$\left\|f_{i}\left(t, y, y^{\prime}, z\right)\right\| \leq c_{i}\left\|y^{\prime}\right\|^{2}+D_{i} \quad$ for all $t \in[0, T]$ and $\left(y, y^{\prime}, z\right) \in \mathcal{D}$, 
then by (3.4)

$$
\left\|F\left(t, y, y^{\prime}, z\right)\right\| \leq c\left(\sum_{i=0}^{r-1} c_{i}\right)\left\|y^{\prime}\right\|^{2}+c\left(\sum_{i=0}^{r-1} D_{i}\right)=\bar{C}\left\|y^{\prime}\right\|^{2}+\bar{D}
$$

and (A2)" is evidently satisfied.

For $(\mathrm{A} 3)^{\prime \prime}$ to be satisfied, by the convexity of $F\left(t, y, y^{\prime}, z\right)$, it is sufficient that for every extremal point $g_{\varepsilon}\left(t, y, y^{\prime}, z\right)$ of $F\left(t, y, y^{\prime}, z\right)$

$$
\left\|F\left(t, y, y^{\prime}, z\right)\right\| \leq 2 \alpha\left(y \cdot g_{\varepsilon}\left(t, y, y^{\prime}, z\right)+\left\|y^{\prime}\right\|^{2}\right)+K
$$

for a.e. $t \in[0, T]$ and all $\left(y, y^{\prime}, z\right) \in \mathcal{D}$.

Now we can state the following.

(3.5) Corollary. Suppose that $f\left(t, y, y^{\prime}, u\right)$ given by (3.1) satisfies the conditions $(\mathrm{A} 1)^{\prime \prime}-(\mathrm{A} 3)^{\prime \prime}$. Then the system (3.2) has a generalized solution in $H^{2}\left([0, T] ; \mathbb{R}^{n}\right)$, i.e. there exists $y \in H^{2}\left([0, T] ; \mathbb{R}^{n}\right)$ which satisfies (3.3).

We wish to emphasize that a generalized solution to (3.2) need not be a classical solution and the verification of this property presents a separate problem. In this example, it is not evident that the solution $y(t)$ intersects transversally the critical levels $\varphi(x)=\alpha_{i}$, and therefore we cannot exclude the possibility that the solution $y(t)$ may remain for a while on one of those levels and for those $t$, because of the discontinuity of the function $f$, the solution to the differential inclusion (3.3) may not satisfy the equation (3.2).

Suppose now that the functions $f_{j}:[0, \infty) \times \mathbb{R}^{n} \times \mathbb{R}^{n} \times \mathbb{R}^{n \cdot m} \rightarrow \mathbb{R}^{n}$, $j=0,1, \ldots, r-1$, are continuous and that

$$
f:[0, \infty) \times \mathbb{R}^{n} \times \mathbb{R}^{n} \times \mathbb{R}^{n \cdot m} \rightarrow \mathbb{R}^{n}
$$

is again defined by (3.1). We choose $R>0$ such that $R>\alpha_{r-1}$ and we introduce the following conditions:

$(\mathrm{B} 1)^{\prime \prime} \quad$ If $\psi(x)>R$ then for every $x^{\prime} \in \mathbb{R}^{n}$ such that $D \psi(x) \cdot x^{\prime}=0$ and every $u$ such that $\|u\| \leq \xi(\psi(x))$ we have

$$
D \psi(x) \cdot f\left(t, x, x^{\prime}, u\right)+D^{2} \psi(x) x^{\prime} \cdot x^{\prime}>0 .
$$

$(\mathrm{B} 2)^{\prime \prime} \quad$ There is a function $\varphi:[0, \infty) \rightarrow(0, \infty)$ such that $s / \varphi(s) \in$ $L_{\mathrm{loc}}^{\infty}[0, \infty), \int_{0}^{\infty}(s / \varphi(s)) d s=\infty$ and $\left\|F\left(t, y, y^{\prime}, z\right)\right\| \leq \varphi\left(\left\|y^{\prime}\right\|\right)$ for a.e. $t \in[0, \infty)$ and all $\left(y, y^{\prime}, z\right) \in \mathcal{D}:=\left\{\left(x, x^{\prime}, w\right):\|x\| \leq \xi(R)\right.$, $\|w\| \leq \xi(R)\}$, where $F\left(t, x, x^{\prime}, z\right)=\left[\underline{f}\left(t, x, x^{\prime}, z\right), \bar{f}\left(t, x, \overline{x^{\prime}}, z\right)\right]$ is defined in the usual way.

(B3)" There exist constants $K, \alpha>0$ such that $\left\|F\left(t, y, y^{\prime}, z\right)\right\| \leq$ $2 \alpha\left(y \cdot w+\left\|y^{\prime}\right\|^{2}\right)+K$ for a.e. $t \in[0, \infty)$ and all $\left(y, y^{\prime}, z\right) \in \mathcal{D}, w \in$ $F\left(t, y, y^{\prime}, z\right)$. 
We consider the problem

$$
\left\{\begin{array}{l}
y^{\prime \prime}(t)=f\left(t, y(t), y^{\prime}(t), \Phi(y)(t)\right) \quad \text { for a.e. } t \in[0, \infty) \\
y(0)=a
\end{array}\right.
$$

where $\Phi(u)=\left(\Phi_{1}(u), \ldots, \Phi_{m}(u)\right)$ is the retardation operator associated with retardations $g_{i}:[0, \infty) \rightarrow \mathbb{R}, g_{i}(t) \leq t, i=1, \ldots, m$. Then we obtain:

(3.7) Corollary. Suppose that $f\left(t, y, y^{\prime}, u\right)$, given by (3.1), satisfies the conditions (B1)"-(B3)". Then the system (3.6) has a generalized solution $y(t)$ in $H_{\mathrm{loc}}^{2}\left([0, \infty) ; \mathbb{R}^{n}\right)$. Moreover, if $\varphi \in L_{\mathrm{loc}}^{\infty}[0, \infty)$ then $y \in$ $W^{2, \infty}\left([0, \infty) ; \mathbb{R}^{n}\right)$.

\section{References}

[1] C. Castaing and M. Valadier, Convex Analysis and Measurable Multifunctions, Lecture Notes in Math. 580, Springer, 1977.

[2] K. C. Chang, The obstacle problems and partial differential equations with discontinuous nonlinearities, Comm. Pure Appl. Math. 33 (1980), 117-146.

[3] J. Dugundji and A. Granas, Fixed Point Theory, Vol. 1, PWN, Warszawa 1982.

[4] J. Duvallet, A theorem of existence for discontinuous differential systems with two point boundary conditions, Nonlinear Anal. 13 (1989), 43-51.

[5] L. H. Erbe and W. Krawcewicz, Nonlinear boundary value problems for differential inclusions $y^{\prime \prime} \in F\left(t, y, y^{\prime}\right)$, this issue, 195-226.

[6] L. H. Erbe and K. Schmitt, On solvability of boundary value problems for systems of differential equations, J. Appl. Math. Phys. 38 (1987), 184-192.

[7] M. Frigon, Application de la théorie de la transversalité topologique à des problèmes non linéaires pour certaines classes d'équations différentielles ordinaires, Dissertationes Math. 296 (1990).

[8] A. Granas, Homotopy extension theorem in Banach spaces and some of its applications to the theory of nonlinear equations, Bull. Acad. Polon. Sci. 7 (1959), 387-394.

[9] A. Granas et Zine el Abdine Guennoun, Quelques résultats dans la théorie de Bernstein-Carathéodory de l'équation $y^{\prime \prime}=f\left(t, y, y^{\prime}\right)$, C. R. Acad. Sci. Paris Sér. I 306 (1988), 703-706.

[10] A. Granas, R. Guenther and J. W. Lee, Nonlinear boundary value problems for ordinary differential equations, Dissertationes Math. 244 (1981).

[11] —, - —, On a theorem of S. Bernstein, Pacific J. Math. 74 (1978), 78-82.

[12] - , - - Nonlinear boundary value problems for some classes of ordinary differential equations, Rocky Mountain J. Math. 10 (1980), 35-58.

[13] J. Hadd ad and J. M. Lasry, Periodic solutions of functional differential inclusions and fixed points of G-selectionable correspondences, J. Math. Anal. Appl. 110 (1983), 295-312.

[14] P. Hartman, Ordinary Differential Equations, Wiley, New York 1964.

[15] T. Kaczyński, Topological transversality and nonlinear equations in locally convex spaces, preprint, 1987.

[16] W. Krawcewicz, Contribution à la théorie des équations non linéaires dans les espaces de Banach, Dissertationes Math. 273 (1988). 
[17] T. Pruszko, Topological degree methods in multivalued boundary value problems, Nonlinear Anal. 5 (9) (1981), 953-973.

[18] - Some applications of the topological degree theory to multivalued boundary value problems, Dissertationes Math. 229 (1984).

[19] C. A. Stuart, Differential equations with discontinuous nonlinearities, Arch. Rational Mech. Anal. 63 (1976), 59-75.

DEPARTMENT OF MATHEMATICS

UNIVERSITY OF ALBERTA

EDMONTON, ALBERTA

CANADA

Reçu par la Rédaction le 2.11.1988

Révisé le 15.5.1989 et le 15.1.1990 